\title{
Critical role of myeloid differentiation factor 88 in necrotizing enterocolitis
}

\author{
Guozhu Yang ${ }^{1,2}$, Pingqian Bao ${ }^{1,2}$, Lie Zhang ${ }^{2,3}$, Zhaoying Lyu², Bin Zhou², Keling Chen², Su Peng ${ }^{2,3}$, Yi Wang ${ }^{1,2}$, Longpei Yao ${ }^{1,2}$, \\ Yin Zhou ${ }^{1,2}$ and Yuan $\mathrm{Li}^{1,2}$
}

BACKGROUND: The importance of Toll-like receptor 4 in necrotizing enterocolitis (NEC) has been intensively studied, but its downstream signaling and the potential regulatory mechanisms remain unidentified. Our study focused on the role of myeloid differentiation factor 88 (MyD88), the first downstream adaptor of Toll-like receptor 4 inflammatory and apoptotic signaling in the pathogenesis of NEC.

METHODS: MyD88 knockout (MyD88-1-Ko) mice and lentivirus-mediated stable MyD88-knockdown cell line (IEC-6) were used. NEC was induced by formula gavage, cold, hypoxia, combined with lipopolysaccharide (LPS) in vivo, or LPS stimulation in vitro. NEC was evaluated by histology and multiple inflammatory cytokines. Enterocyte apoptosis was evaluated by terminal-deoxynucleoitidyl transferase-mediated nick end labeling (TUNEL) or Annexin analysis. Inflammatory or apoptotic molecules including NF-KB, Tol//L-1R domain-containing adaptor-inducing IFN- $\beta$, interferon regulatory factor $3, \mathrm{Bax}, \mathrm{BCl}$ 2 , and caspases were examined by quantitative real-time PCR (qRT-PCR).

RESULTS: In the MyD88-Ko group, NEC severity and intestinal enterocyte apoptosis rate were reduced, the expression of $N F-K B$, caspases, and Bax, were all downregulated, while Toll/ IL-1R domain-containing adaptor-inducing IFN- $\beta$ and interferon regulatory factor 3 were upregulated, and antiapoptotic gene $B C l-2$ remained stable. Cytokine levels of interleukin (IL)-6, IL-1 $\beta$, and tumor necrosis factor-a (TNF-a) were also all decreased.

CONCLUSION: MyD88-dependent signaling is the prevailing inflammatory and apoptotic signaling in Toll-like receptor 4 downstream signaling; MyD88-Ko resulted in reduced inflammatory severity and apoptosis, though MyD88-independent signaling can also be activated, but is of less dominant for the development of NEC.

N ecrotizing enterocolitis (NEC) is the leading cause of death from gastrointestinal disease in preterm infants, and remains as a major cause of long-term dysfunction in those who survive the disease (1-4). Although the pathogenesis of NEC remains incompletely understood, emerging evidence suggests that Toll-like receptors (TLRs), especially TLR4, plays an essential role in innate and adaptive immune responses to invading pathogens in the development of NEC (5-10). Upon recognition of a specific pathogenic ligand, signaling cascades are activated through several adaptor molecules, which determine the specificity of the response (11). The first and mostly essential utilized adaptor is myeloid differentiation factor 88 (MyD88), which was originally described as part of the interleukin (IL)-1 receptor-signaling complex. MyD88 is almost required in all TLRs signaling (12-14). Upon stimulation, two modes of the signaling will be initiated, one of them is TLRs signals via the adaptor protein MyD88 (MyD88-dependent pathway) $(15,16)$. The adaptor molecule MyD88 is necessary for TLRs signaling events, which results in the induction of a core set of responses through the activation of nuclear factor- $\mathrm{KB}(\mathrm{NF}-\mathrm{\kappa B})$ and the production of proinflammatory cytokines; a second signaling route for TLRs was involved to the adaptor proteins Toll/IL-1R domain-containing adaptor-inducing IFN- $\beta$ (TRIF $(17,18)$; MyD88-independent pathway), the signaling of TLRs occurs through the TRIF-dependent pathway (also called MyD88independent pathway) that leads to the activation of interferon regulatory factor 3 (IRF3) - a key transcription factor that is responsible for the induction of IFN genes (19-22). TRIF and IRF-3 are all to be responsible for the MyD88-independent induction of NF- $\mathrm{\kappa B}$ as well. The transcription factor NF- $\kappa \mathrm{B}$ lies at the nexus of both antiapoptotic and proinflammatory cascades in chronic inflammatory bowel disease (23-25).

The regulation of enterocyte apoptosis in the newborn intestine plays an important role in determining the balance between mucosal homeostasis and disease (26). Such regulation may be of particular relevance to the development of NEC, a devastating illness of premature neonates that is characterized by exaggerated enterocyte apoptosis leading to severe intestinal injury. Lipopolysaccharide (LPS) can induce apoptosis via a death pathway involving TLR4 signaling. MyD88 subsequently binds Fas-associated death domain-containing protein, which promotes activation of caspase 8 (27-29). Caspase 9 is related to the mitochondrial membrane disruption that is another important process controlling apoptosis. Caspase 8 and caspase 9 are both 
activated in the apoptotic process and subsequently processes executioner caspases $3(28,30)$. These steps are all essential for apoptosis induction.

In seeking to understand the increased susceptibility of the preterm infant to the development of NEC, we now focus on the function of MyD88 within the developing intestine and examine the relative roles of TLR4/MyD88-dependent and TLR4/MyD88-independent signaling in the neonatal intestinal inflammatory response and apoptosis mechanism. We now demonstrate that NEC is associated with the activation of TLR4/MyD88-dependent signaling and TLR4/MyD88independent signaling. Taken together, these findings provide evidence that MyD88 knockout can significantly reduce the severity of inflammation and apoptosis in NEC that suggests a mechanism to explain the injurious effects of LPS on the intestinal epithelium in the pathogenesis of NEC.

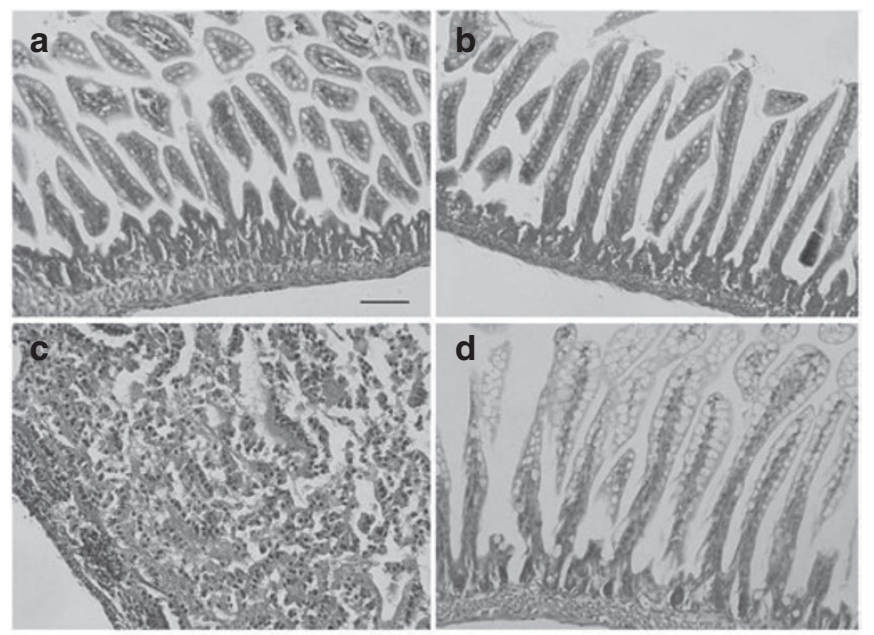

e

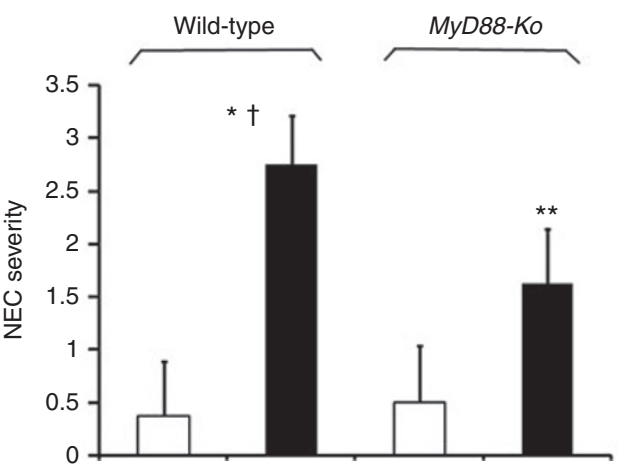

Figure 1. MyD88-Ko mice were protected from the development of NEC. Hematoxylin and eosin staining of intestinal mucosa structure in the different groups. (a) MyD88-WT or (b) MyD88-Ko served as breast fed controls. NEC was induced in newborn (c) MyD88-WT and (d) MyD88-Ko mice (original magnification: $\times 400$, bar $=50 \mu \mathrm{m}$ ). NEC severity was quantified by pathological scoring system (e). No fill, control; black fill, NEC. ${ }^{*} P<0.05$ by ANOVA compared with MyD88-Ko with NEC, ${ }^{+} P<0.05$ by ANOVA compared with MyD88-WT breast fed control animals. ${ }^{* *} P<0.05$ by ANOVA compared with MyD88-Ko breast fed control animals. Data from four separate experiments ( $n=8$ animals/experimental group). Ko, knockout; NEC, necrotizing enterocolitis; WT, wild type.

\section{RESULTS}

NEC Severity Was Attenuated in $M y D 88^{-1-}$ Animal Group

In the current research, all the animals in four groups are survived and the incidences of NEC in wild type (WT) and MyD88-Ko animals exposed to NEC are $100 \%$ (8/8) and $62.5 \%$ (5/8), respectively. Compared with breastfeeding control mice, WT littermates showed marked disruption in mucosal structure after induction of NEC. The inflammatory severity of NEC was significantly reduced in $M y D 88-K o$ mice compared with WT as evaluated by histology $(P<$ 0.05). There were no statistical differences regarding intestinal histology between $M y D 88-K o$ and WT control group $(P>0.05)$, which were breast fed alone (Figure 1). Intestinal inflammation severity scores are shown in Supplementary Table S1 online.

\section{MyD88 Knockdown in IEC-6 Cell Line}

$M y D 88$ expression was analyzed by quantitative real-time PCR (qRT-PCR) and western blot in MyD88-knockdown intestinal enterocyte cells (IEC-6). Compared with control and nontarget-treated cells, MyD88 expression was effectively silenced in two LvshMyd88 cell lines by 79.3 and $72.6 \%$, respectively (Figure 2a). MyD88 protein level was significantly reduced in two silences cell lines, which was consistent with the result of qRT-PCR (Figure 2b).

\section{Greater Difference of MyD88 Expression Was Found Between Knockdown and WT Cell Lines After LPS Stimulation}

LPS was administrated as inflammatory stimulator in MyD88-knockdown and WT IEC-6 cell lines. The mRNA and protein level of MyD88 was evaluated by qRT-PCR and western blot. After stimulation, MyD88 mRNA expression was significantly upregulated at all selected time points in IEC-WT group, while it remained stable at low level in IEC-MyD88-knockdown group (Figure 2c). Comparable difference of MyD88 protein level was also detected between IEC-WT and IEC-MyD88-knockdown cell lines and it was consistent with the result of qRT-PCR (Figure 2d).

\section{The Apoptosis of Enterocytes Is Inhibited in Both $M y D 88^{-/-}$Mice and MyD88-Knockdown Cell Strain}

The apoptosis rate of enterocytes in different groups was evaluated by Annexin V analysis. Before LPS treatment, apoptosis rate was lower in MyD88-knockdown cell lines $(0.68 \pm 0.07)$ than that in WT cell line $(2.43 \pm 0.67)$. After LPS treatment, the enterocyte apoptotic rate was significantly increased in IEC-6 WT cells $(11.76 \pm 1.65)$, but it remained stable in $M y D 88$-knockdown group $(0.34 \pm 0.02)$. The apoptosis rate of $M y D 88$-knockdown group was significantly lower than that in WT group after LPS stimulation (Figure 3). Apoptotic ratio before and after LPS treatment is shown in Supplementary Table S2 online.

The effects of TLR4/MyD88 activation on enterocyte apoptosis were assessed by terminal deoxynucleoitidyl transferasemediated nick end labeling (TUNEL) in small intestinal tissue 
a

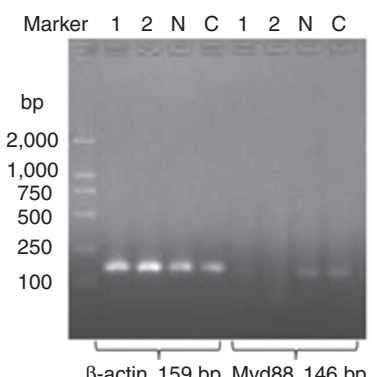

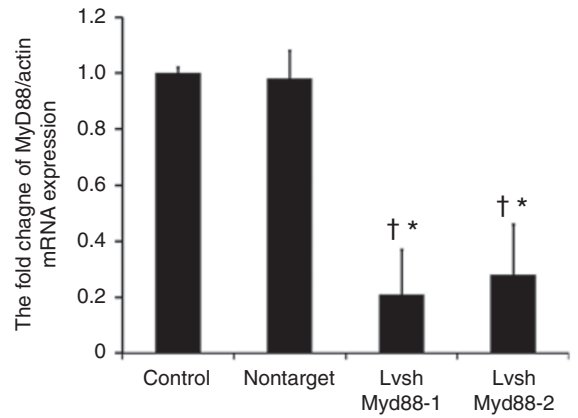

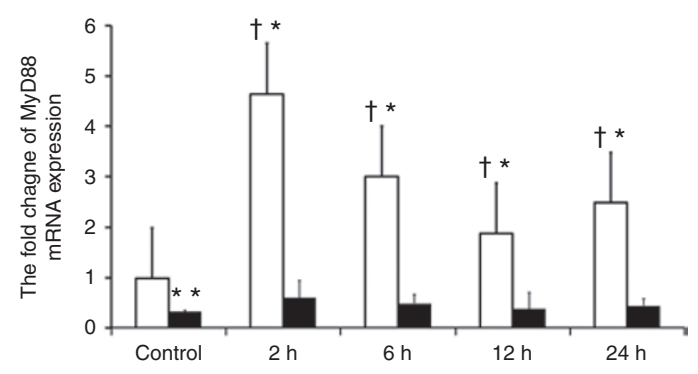

b

d
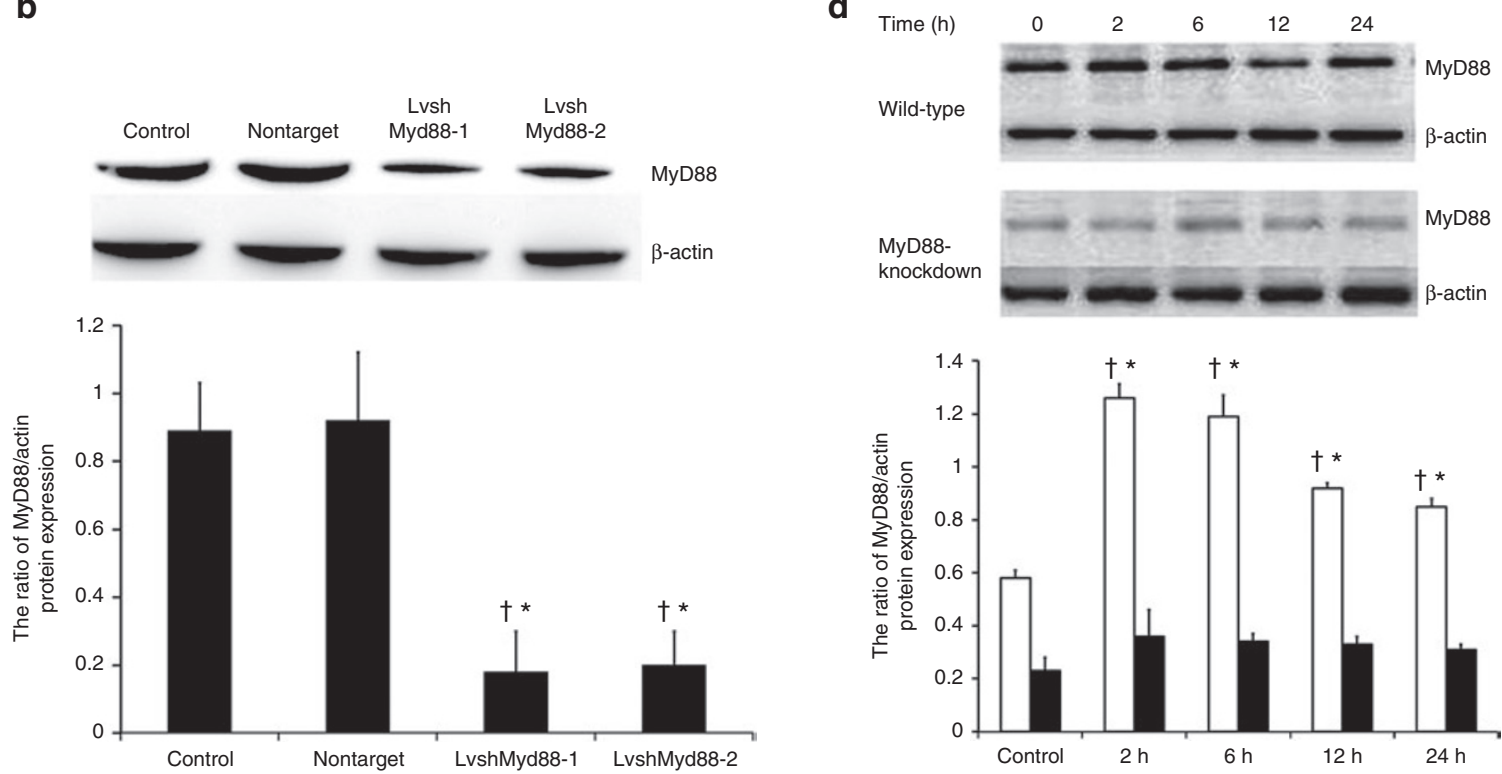

Figure 2. MyD88 expression in vivo before and after stimulation of LPS. (a) Quantitative real-time PCR (qRT-PCR) or (b) western blot assay MyD88 mRNA expression and protein expression in the cells after lentivirus transduction. Control: untreated IEC-6 group; nontarget: IEC-6 transfected with vector only; LvshMyD88- 1, 2: two IEC-6 lines transfected with lentiviral plasmid that expressed the shRNA of MyD88. Quantification of MyD88 mRNA (a, right) and protein (b, bottom) expression was analyzed. ${ }^{*} P<0.05$ by ANOVA compared with control group. ${ }^{+} P<0.05$ by ANOVA compared with nontarget group. After stimulation of LPS $(50 \mu \mathrm{g} / \mathrm{ml})$ at different times, MyD88 expression was detected by qRT-PCR or western blot (d, top) in IEC-wt and IEC-MyD88-knockdown group. Quantification of (c) MyD88 mRNA and (d) protein (bottom) expression was analyzed. ${ }^{*} P<0.05$ by two-tailed Student's $t$-test compared with IEC6-wt control (no stimulation with LPS). ${ }^{*} P<0.05$ by ANOVA compared with IEC-MyD88-knockdown with LPS at different time. ${ }^{\dagger} P<0.05$ by ANOVA compared with IEC-wt control group (no fill: IEC-WT; black fill: IEC-MyD88-knockdown). IEC, intestinal enterocyte cells; LPS, lipopolysaccharide; WT, wild type.

of newborn mice with NEC. A marked decrease of enterocyte apoptosis was detected in MyD88-Ko mice compared with WT mice $(P<0.05)$, there was no difference of enterocye apoptosis between breast fed control of two strain mice groups $(P>0.05$; Figure 4).

\section{NF-KB Activation Was Decreased in MyD88 $8^{-/-}$Mice and \\ Potentially Mediated by TRIF-Dependent Signaling}

The mRNA expression of the transcription factor NF- $\kappa B$ was detected by qRT-PCR and there was no difference between controls from either WT or MyD88-Ko. While after NEC induction, the expression of $N F-\kappa B$ is significantly decreased in MyD88-Ko mice compared with WT mice. TRIF and IRF-3 as key genes in TLR4/MyD88-independent signaling, both were upregulated in MyD88-Ko mice than those in WT mice; MyD88-WT mice with NEC demonstrated upregulated and higher in the level of TRIF and IRF-3 expression than control (Figure 5).

\section{MyD88 Gets Involved in the Regulation of Apoptosis}

The expression of key apoptosis related genes, including caspase 8, 9, 3, Bax and Bcl-2 were evaluated by qRT-PCR. In MyD 88 -Ko NEC groups, caspase 3 and caspase 8 were decreased compared with WT NEC groups, but were all increased compared with breast fed control. The expression of caspase 9 in MyD88-Ko NEC groups was more decreased compared with WT NEC groups, while it has no difference compared with $M y D 88-K o$ breast fed control. Besides, compared with breast fed control, the expression of Bax remained unchanged in MyD88-Ko mice but increased significantly in WT mice after induction of NEC; the expression of $\mathrm{Bcl}-2$ remained stable within two mice strains with NEC or breast fed controls (Figure 5).

\section{MyD88-Knockdown Attenuates the Production of} Proinflammatory Cytokines In Vitro and In Vivo

Cytokine level of IL- 6 and IL-1 $\beta$ in cell supernatant were examined by Liquid chip (Luminex, Austin, TX). Compared 


\section{Articles | Yangetal.}

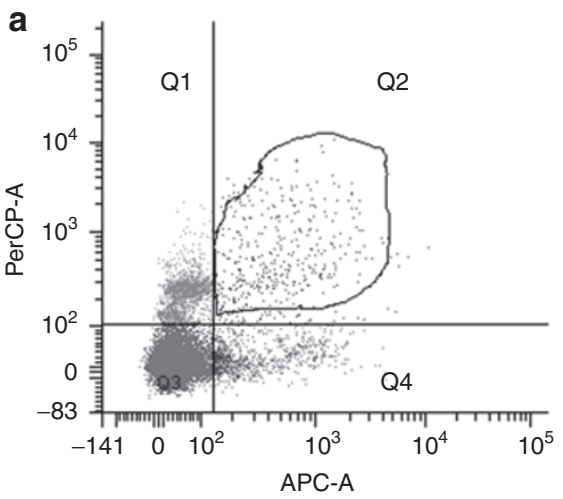

C

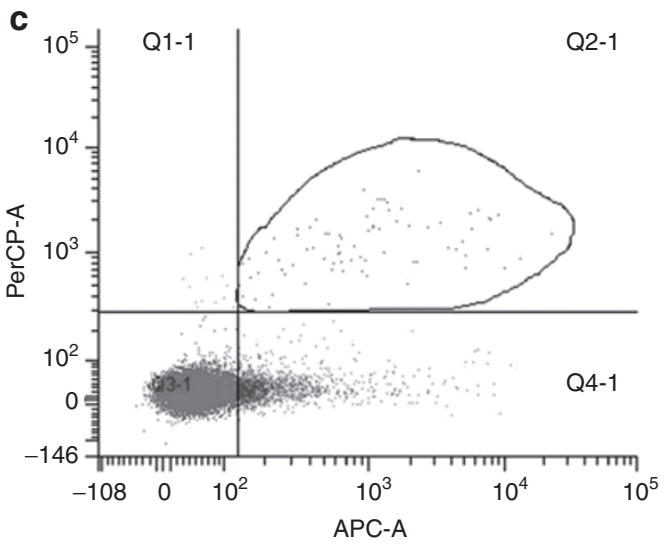

e

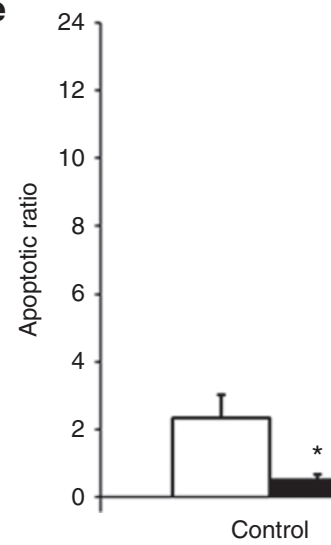

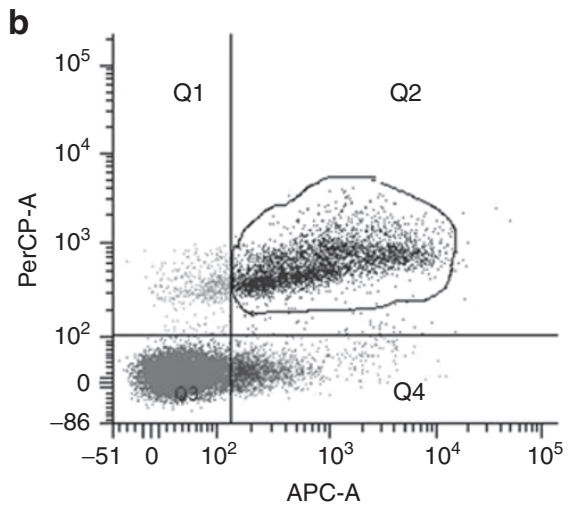
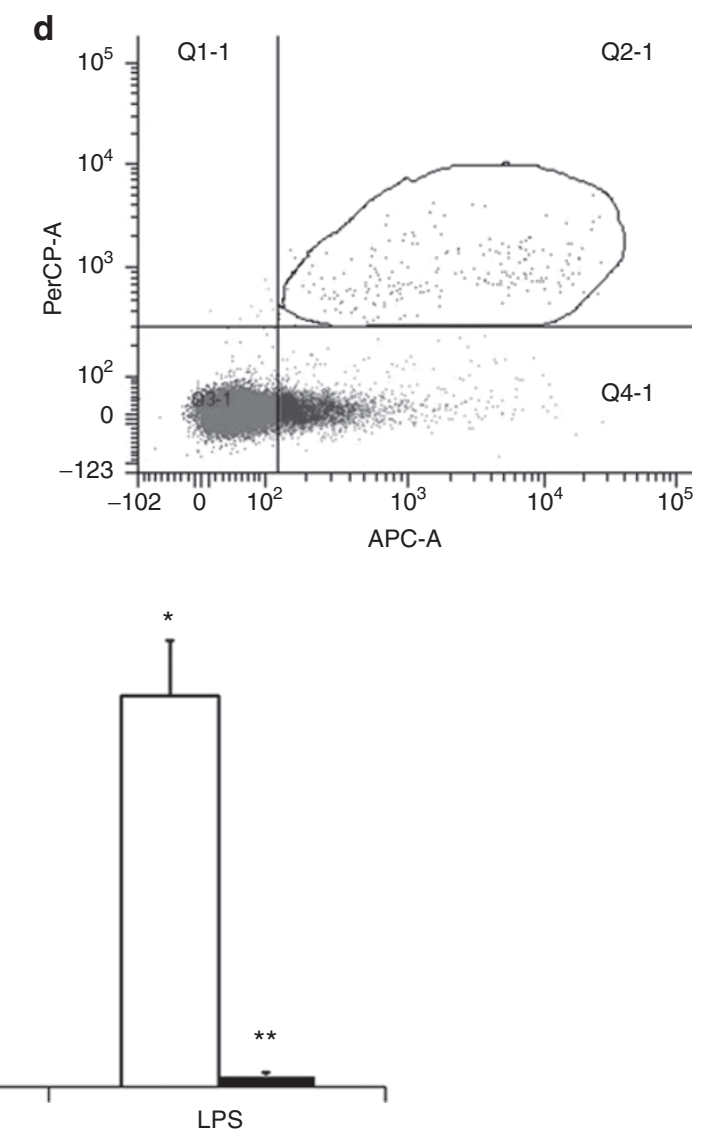

Figure 3. FCM detection of apoptosis after treated with LPS in vitro. (a) IEC-WT control group; (b) $12 \mathrm{~h}$ after stimulation with LPS in IEC-WT group; (c) IEC-MyD88-knockdown control group; (d) $12 \mathrm{~h}$ after stimulation with LPS in IEC-MyD88-knockdown group; the Q2 quadrant (annexin V+/7-AAD+) indicates the percentage of apoptosis. (e) Quantification of apoptosis rate determined by a blinded observer (no fill: IEC-WT; black fill: IEC-MyD88knockdown). ${ }^{*} P<0.05$ by ANOVA compared with IEC-6-wt control, ${ }^{* *} P<0.05$ by ANOVA compared with IEC-6-WT with LPS. Representative data from four separate experiments with three times per group. IEC, intestinal enterocyte cells; LPS, lipopolysaccharide; WT, wild type.

with nontreated control cells, both IL-6 and IL-1 $\beta$ level were increased after LPS treatment in IEC-6 cells. However, the level of IL- 6 and IL- $1 \beta$ in IEC-6-MyD88-knockdown cells remained stable at all time points after LPS stimulation (Figure 6a,b).

In WT mice with NEC, IL- $1 \beta$, and tumor necrosis factor- $\alpha$ $($ TNF- $\alpha$ ) level in intestinal tissues were both higher than those of breast fed groups. In $M y D 88-K o$ mice, IL- $1 \beta$ level was increased but no difference was detected for TNF- $\alpha$ compared with breast fed mice; both IL- $1 \beta$ and TNF- $\alpha$ level in tissue were lower in $M y D 88$-Ko mice with NEC compared with WT NEC groups (Figure 6c,d).

\section{DISCUSSION}

In our study, we successfully provided evidence that $M y D 88-K o$ animals and $M y D 88$-knockdown intestinal enterocyte cells lead to decreased severity of NEC compared with WT groups. We also detected the proinflammatory cytokines level of IL-1 $\beta$ and IL-6 in cultured MyD88-knockdown cell supernatant and the level of IL- $1 \beta$ and TNF- $\alpha$ in MyD88-Ko animal intestinal tissue. Our results also showed the deficiency of $M y D 88$ resulted in decreased proinflammatory cytokines, which could further confirm our previous conclusion of pathological 

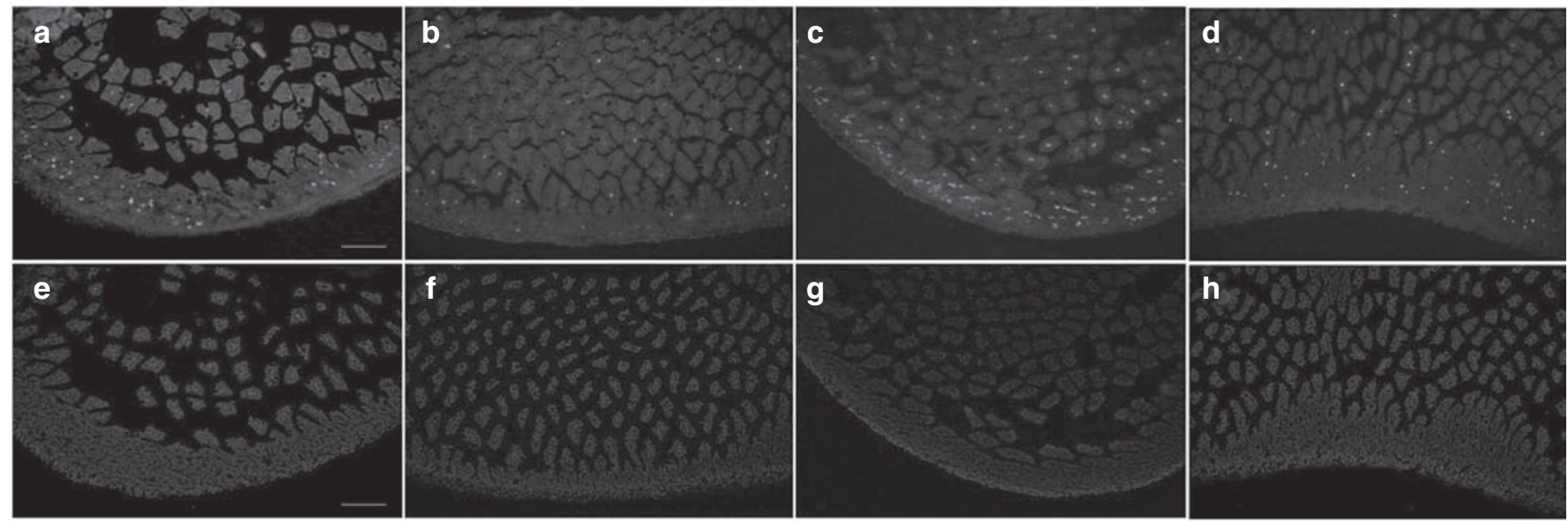

g

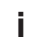

i
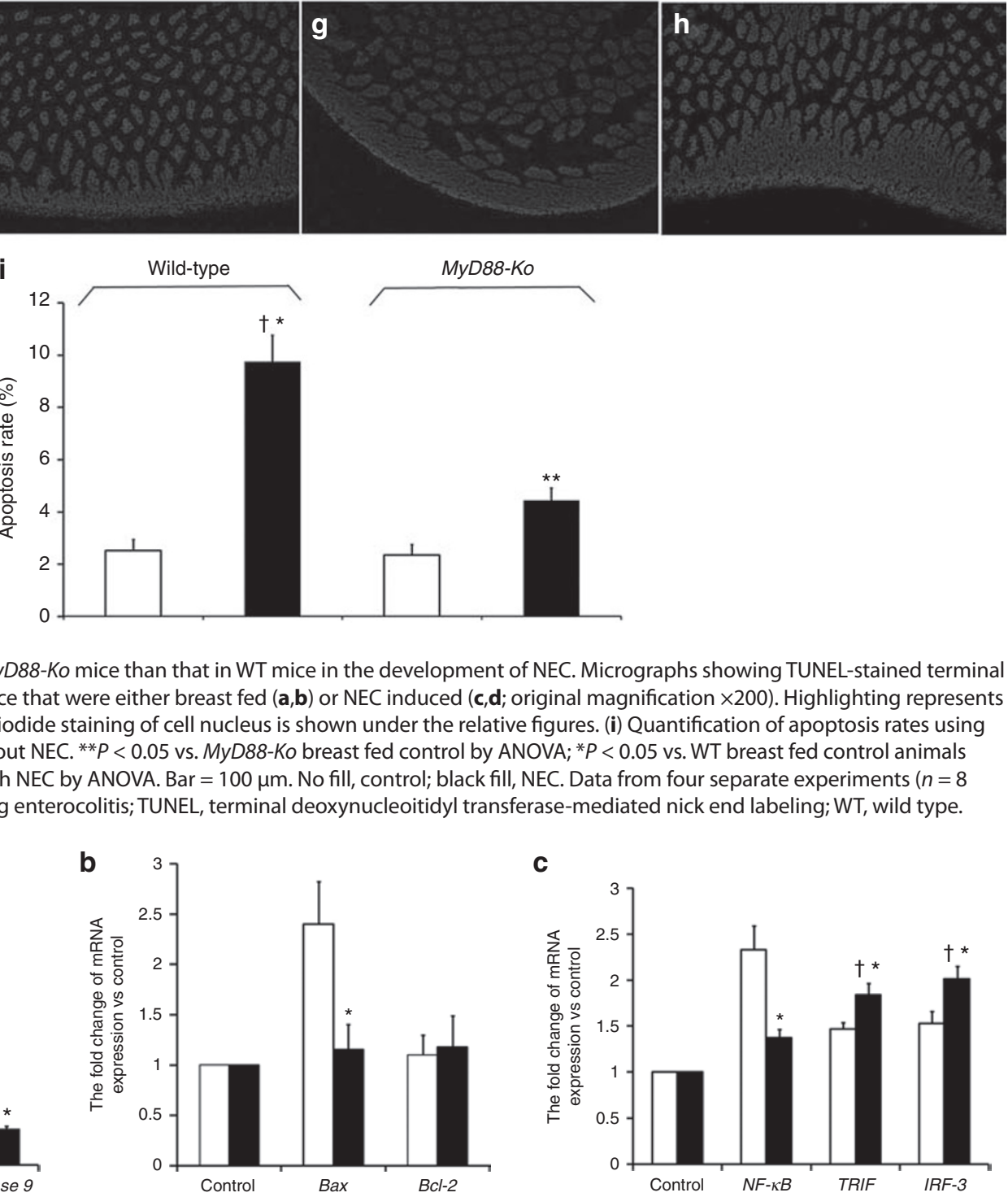

$y D 88-K_{0}$

Figure 4. Enterocyte apoptosis was less in MyD88-Ko mice than that in WT mice in the development of NEC. Micrographs showing TUNEL-stained terminal ileum of $(\mathbf{a}, \mathbf{c})$ WT mice and $(\mathbf{b}, \mathbf{d})$ MyD88-Ko mice that were either breast fed $(\mathbf{a}, \mathbf{b})$ or NEC induced $(\mathbf{c}, \mathbf{d}$; original magnification $\times 200)$. Highlighting represents TUNEL-positive enterocytes. (e-h) Propidium iodide staining of cell nucleus is shown under the relative figures. (i) Quantification of apoptosis rates using Image J in WT or MyD88-Ko mice with or without NEC. ${ }^{*} P<0.05$ vs. MyD88-Ko breast fed control by ANOVA; ${ }^{*} P<0.05$ vs. WT breast fed control animals by ANOVA; ${ }^{\dagger} P<0.05$ vs. MyD88-Ko animals with NEC by ANOVA. Bar $=100 \mu \mathrm{m}$. No fill, control; black fill, NEC. Data from four separate experiments $(n=8$ animals/experimental group). NEC, necrotizing enterocolitis; TUNEL, terminal deoxynucleoitidyl transferase-mediated nick end labeling; WT, wild type. a

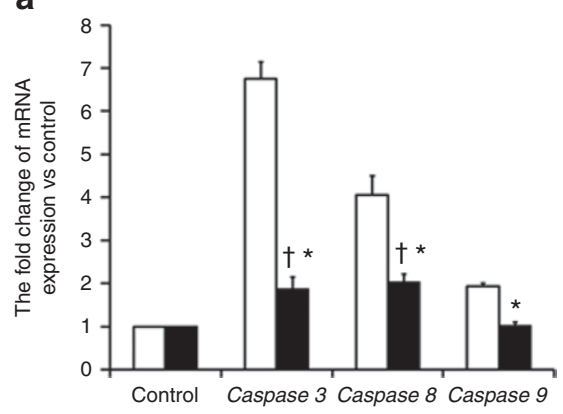

b

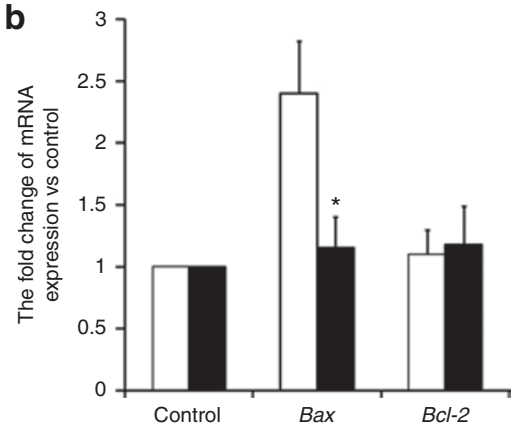

C

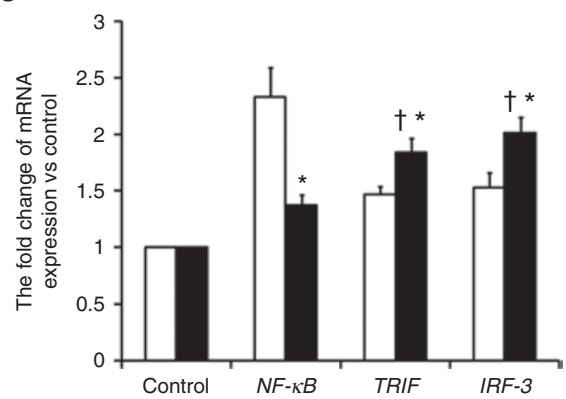

Figure 5. Analysis of gene expression in apoptosis and TLR4 signaling. Quantitative real-time PCR (qRT-PCR) showing (a) caspase 3, caspase 8, caspase 9, (b) Bax, BCl-2, (c) NF- KB, TRIF, and IRF-3 in WT and MyD88-Ko mice with NEC compared to breast fed control. RT-PCR data are normalized to $\beta$-actin RNA expression with $2^{-\Delta \Delta C t}$ calculated comparing to control, analyzed by ANOVA, ${ }^{*} P<0.05$ vs. WT NEC groups, ${ }^{+} P<0.05$ vs. MyD88-Ko breast fed control groups. No fill, WT; black fill, MyD88-Ko; four separate experiments with eight mice per group. IRF, interferon regulatory factor; NEC, necrotizing enterocolitis; TRIF, Toll/IL-1R domain-containing adaptor-inducing IFN- $\beta$; WT, wild type.

evaluation. Not only that, we also demonstrated that the deficiency of MyD88 could protect from increasing apoptosis of intestinal epithelial cells. These findings indicate that MyD88 is a key factor in attenuating intestinal enterocyte apoptosis and avoiding the development of NEC.

NEC develops in a stressed preterm infant in the setting of intestinal barrier disruption, systemic inflammation and leads to multisystem organ failure. The intestinal barrier lies at the interface between microbes within the intestinal lumen and the immune system of the host, and has both immunological and mechanical components. These components serve to protect the host from invading pathogens. Some factor, such as the components of Gram-negative bacteria, can specifically recognize TLRs at the intestinal interface and activate TLRs-MyD88 signaling that leads to the apoptosis of enterocytes. Apoptosis is a form of programmed cell death that allows elimination 
a

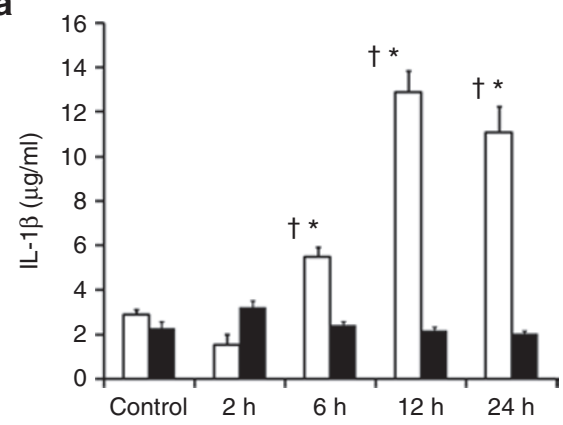

C

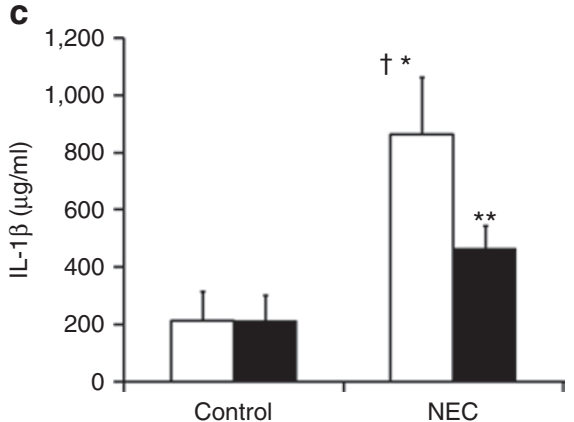

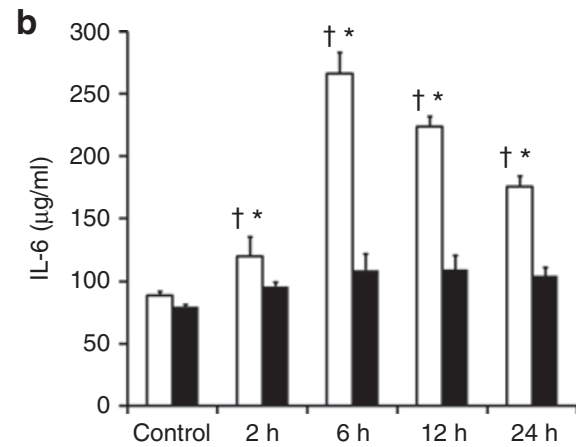

d

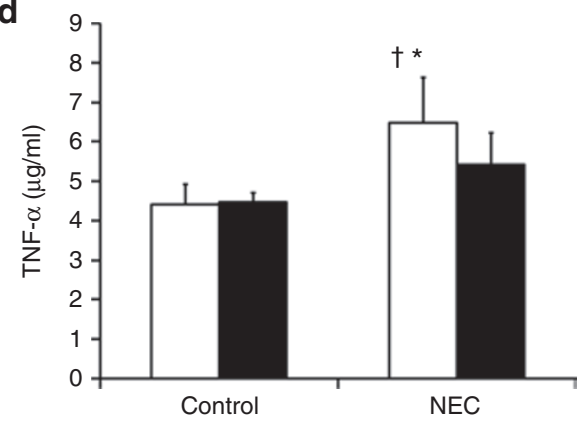

Figure 6. Cytokine detection in vivo and vitro. Cytokine levels of (a) IL-1 $\beta$ and (b) IL- 6 in cell supernatant at different times after LPS treatment; ${ }^{+} P<0.05$ vs. MyD88-knockdown with LPS at the time by ANOVA; ${ }^{P} P<0.05$ vs. WT breast fed control by ANOVA. Representative data from four separate experiments with three times per group. No fill, WT; black fill, MyD88-knockdown strain. The level of (c) IL-1 $\beta$ and (d) TNF- $\alpha$ in intestinal tissue from MyD88 WT

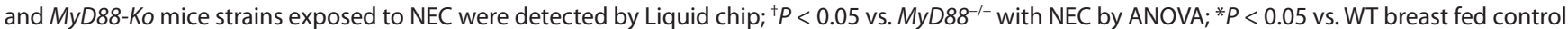
by ANOVA. ${ }^{* *} P<0.05$ vs. MyD88-Ko breast fed control by ANOVA; data from four separate experiments ( $n=5$ animals/experimental group). No fill, WT; black fill, MyD88-Ko mice. IL, interleukin; NEC, necrotizing enterocolitis; TNF, tumor necrosis factor; WT, wild type.

of irreversibly damaged cells while preserving overall tissue function, and it plays a vital role in normal developmental processes. However, overwhelming activation of apoptosis may result in tissue failure. The impairments in the function of the intestinal barrier may predispose the host to the invasion of gut-derived microbes and to the development of systemic inflammatory disease. This process, termed "bacterial translocation," may be compounded during instances in which the mechanisms that regulate the repair of the intestinal barrier are disrupted. In the results of our study, the deficiency of MyD88-Ko can lead to decreased apoptosis of enterocytes in NEC occurrence. We demonstrate that the deficiency of $M y D 88$ have attenuated NEC inflammatory severity because of protecting from increased apoptosis and "bacterial translocation" in intestinal barrier.

LPS binding to TLR4, triggers activation of the cellular signaling pathways resulting in nuclear translocation of NF- $\mathrm{\kappa B}$ and apoptosis. The molecular mechanism that links the NF- $\kappa \mathrm{B}$ signaling to the recruitment and activation of caspases remains unclear. TLR4 and its respective intracellular binding partners, MyD88, TRIF, along with the extrinsic Fas-associated death domain - caspase 8 pathway, have been shown to mediate LPSinduced apoptosis. However, questions remain as to how this signaling pathway activates the effector proteases of apoptosis $(31,32)$. It has been suggested that members of the Bcl-2 family play the role of mediator in LPS-induced apoptosis. LPS upregulates the expression of the proapoptotic Bcl-2 family members Bax, and downregulates the levels of the antiapoptotic members, such as Bcl-2 $(33,34)$. We next attempted to find the change of apoptosis associated gene among the different groups in our study. Our results show that in the MyD88-Ko NEC groups, caspase 3, caspase 8, caspase 9, and Bax were all decreased compared with WT NEC groups, but not $B c l-2$. In MyD88-Ko mice with NEC group, the expression of caspase 8 and caspase 3 were both higher than in the MyD88-Ko control group. However, there was no difference in caspase 9 and $\mathrm{Bcl}$ 2 expression between $M y D 88$-Ko mice with or without NEC. Taken together, we infer that $M y D 88$ is both related to the Fasassociated death domain-caspase 8 pathway and caspase 9 pathway gene expression, Bax-mediated apoptosis regulation is also regulated by $M y D 88$. However, when treated with LPS in MyD88-Ko groups, caspase 9 and bax did not play a part in apoptosis regulation but the TLR4/MyD88-independent signaling can also be activated and TRIF-mediated the Fasassociated death domain-caspase 8 pathway can also lead to apoptosis in intestinal epithelial cells, but it is not relevant with caspase 9 and Bax regulation.

Given these findings, TLR4 downstream mechanism involved in NEC was deeply studied and the result showed that the expression of NF- $\kappa B$ was decreased in MyD88-Ko mice with NEC compared with $M y D 88-W T$ mice with NEC, but still greater than MyD88-Ko mice without NEC. It was interesting to note that, in the MyD88-Ko mice with NEC, the molecular of TRIF and IRF-3 that express in the TLR4/MyD88-independent signaling was increased compared with the MyD88-WT mice with NEC. TLR4 triggers at least two intracellular signaling pathways, one 
mediated by the common TLR adaptor MyD88 and one that relies on TRIF. The different intracellular adaptors of TLR4 lead to different ways of cell activation; MyD88 activation leads to early phase $N F-\kappa B$ activation, among others, IL-1R-associated kinase and TNFR-associated factor-6, whereas activation of TRIF also leads to NF- $\mathrm{KB}$ activation, but with delayed kinetics (35). Knowledge of the relative roles of the MyD88-dependent and MyD88-independent pathways of TLR4 signaling in host defense against pathogens is highly limited. Johnson et al. (36) showed that MyD88 exerts its negative regulatory effects upon the TRIF-dependent pathway and this may be an epithelial cellrestricted event, because they did not detect similar regulatory pathways within macrophages. This is consistent with our findings in intestinal epithelial cell. It will be interesting to study which part of the immune system is responsible for the increased inflammation in the MyD88-Ko group. Possible MyD88-Koindependent mediators include complement and $\mathrm{Ab}$ responses and the TRIF pathway of TLR4 signaling.

In conclusion, based on MyD88-Ko mice and a lentivirusbased RNA interference for Myd88 in intestinal epithelial cell lines, our results demonstrate that, with the activation of LPS, the crucial TLR4/MyD88-dependent signaling is activated, which results in releasing proinflammatory and proapoptotic cytokines, so that the destruction of the intestinal mucosa followed by the development of NEC. MyD88 knockout significantly reduce the severity of inflammation and apoptosis in NEC, though MyD88-independent signaling can also be activated, but is of less dominant for the development of NEC. Our findings shed new light on the effects of MyD88-dependent TLR4 signaling is crucial in the innate immune response of intestinal inflammatory disease.

\section{METHODS}

\section{Animals, Cells, and Reagents}

Six- to ten-wk-old gender-matched MyD88-Ko mice and matched MyD88-WT mice were purchased from Model Animal Research Center of Nanjing University, Nanjing, China. Mice were kept in specific pathogen free conditions and fed by free access to a standard diet and water. Eight newborn mice from WT or MyD88-Ko mice at postnatal day 10 were separated from mothers and housed in a selfmade newborns incubator to be NEC induced. As control groups, another eight newborn mice from WT or MyD88-Ko mice were still kept with their mothers to feed with breast milk. All animal experiments were conducted according to the guidelines of Animal Use and Care Committee at Sichuan University, and executed according to the National Animal Welfare Law of China.

IEC-6 and 293T cells were obtained from American Type Culture Collection and cultured in Dulbecco's modified Eagle's medium high glucose medium (Sigma, St Louis, MO) with 10\% fetal bovine serum, L-glutamine $(0.1 \mathrm{mg} / \mathrm{ml})$, and antibiotics $(100 \mathrm{U} / \mathrm{ml}$ penicillin and $0.1 \mathrm{mg} / \mathrm{ml}$ streptomycin) at $37^{\circ} \mathrm{C}$ in a humidified atmosphere containing $5 \% \mathrm{CO}_{2}$.

\section{Experimental NEC and Sampling}

NEC was induced in 10-d-old MyD88-Ko or WT strains by formula gavage (Similac Advanced infant formula: Esbilac canine milk replacer 2:1) five times daily for $3 \mathrm{~d}$. Components comparison between mouse milk and formula gavage is shown in Supplementary Table S3 online. Animals are fed with $200 \mu \mathrm{g} / 5 \mathrm{~g}$ of mouse body weight by gavage over 2-3 min, using a 24 -French tubular needle which was placed into the mouse esophagus under direct vision. All the NEC mice are exposed to intermittent hypoxia $\left(5 \% \mathrm{O}_{2}, 95 \% \mathrm{~N}_{2}\right)$ for $10 \mathrm{~min}$ using a modular hypoxic chamber and cold asphyxia stress $\left(10 \mathrm{~min}, 4^{\circ} \mathrm{C}\right)$ three times daily for $3 \mathrm{~d}$. LPS is fed separately by $2 \mathrm{mg} / \mathrm{kg}$ of mouse body weight daily. Control animals of both strains were breast fed. Animals then were killed and samples were obtained, the terminal ileum was harvested $1 \mathrm{~cm}$ proximal to the ileocecal valve in $10 \%$ neutral buffered formalin paraffin-embedded, sectioned, and stained with H\&E; other intestinal were frozen in liquid nitrogen for RNA collection and cytokine measurement.

The cultured cells were divided into $2,6,12$, and $24 \mathrm{~h}$ groups. Experimental groups were treated with $50 \mu \mathrm{g} / \mathrm{ml} \mathrm{LPS}$. Control was set for each experimental group and treated with phosphate-buffered solution (PBS). After stimulation, we collected the cells of every group for RNA or protein isolation and the supernatant simultaneously for the detection of downstream cytokines. Each sample was analyzed in triplicate.

\section{Pathological Evaluation}

The severity of experimental NEC was evaluated based on a validated scoring system, which quantitatively determines the severity from 0 (normal) to 3 (severe (37)). The definition for each histological grade was as follows: (0), normal (1), mild, separation of the villus core, without other abnormalities; (2), moderate, villus core separation, submucosal edema, and epithelial sloughing; (3), severe, denudation of epithelium with loss of villi, full thickness necrosis, or perforation. NEC was thought to be induced when the score was $\geq 2$. Pathological evaluation was done by two pathologists in a blind fashion.

\section{TUNEL}

TUNEL assay was performed using the in situ cell death detection kit (Roche, Mannheim, Germany), according to the manufacturer's instructions. Dewaxed, rehydrated paraffin sections $(4 \mu \mathrm{m})$ of intestinal tissue were washed in PBS and digested with $20 \mu \mathrm{g} / \mathrm{ml}$ Proteinase $\mathrm{K}$ (Boehringer, Mannheim, Germany) for $20 \mathrm{~min}$ at $37^{\circ} \mathrm{C}$. Following washing in PBS, sections were incubated with TUNEL reaction mixture containing TdT and fluorescein-dUTP, at $37^{\circ} \mathrm{C}$ for $60 \mathrm{~min}$, followed by extensive washing. Finally, a drop of PBS was added to the sections before they were analyzed under a fluorescence microscope (Olympus, Tokyo, Japan). Images of the tissue were obtained using an OlympusDD70BX51 image acquisition system (Olympus).

\section{Lentivirus-Mediated RNA Interference for MyD88}

Sequences with $100 \%$ homology to regions within the open reading frame of rat Myd88 (NM_198130 in GENBANK) were selected using the siRNA Selection Program of Whitelead institute. The obtained sequence 5'-AACAGACAGACTATCGGCTTA-3' was used to generate oligonucleotide pairs, which were then annealed and ligated into the pPGK-puro-CMV-tGFP vector (Sigma), producing a transfer plasmid (pLVshMyD88) that expressed the shRNA of MyD88. The pLVshMyD88, pCMV-dR8.2 dvpr (Sigma), and pCMV-VSVG (Sigma) were cotransfected into 293T cells using the method of calcium phosphate precipitation. The produced lentiviral particles were harvested at $72 \mathrm{~h}$ after transfection, filtered through a $0.45 \mu \mathrm{m}$ filter (Millipore, Westborough, MA), and kept at $-80^{\circ} \mathrm{C}$. The transduction of IEC- 6 was

Table 1. Sequences of PCR primers and probe

\begin{tabular}{lll}
\hline Gene & \multicolumn{1}{c}{ MyD88 } & \multicolumn{1}{c}{$\beta$-actin } \\
\hline Upstream & 5'-CTAGCCTTGTTAGACCGTGA-3' $^{\prime}$ & 5'-CGTGAAAAGATGACCCAGAT-3' $^{\prime}$ \\
Downstream & $5^{\prime}$-GTCTGTGGGACACTGCTCT-3' & 5'-ACCCTCATAGATGGGCACA-3' $^{\prime}$ \\
Taqman & $5^{\prime}$-FAM-AGGAGGACTGCCAGAAATACATA-3' & 5'-FAM-TCAACACCCCAGCCATGTACGT-TAMRA-3' $^{\prime}$ Length \\
\hline
\end{tabular}


Table 2. Sequences of $P C R$ primers

\begin{tabular}{|c|c|}
\hline Gene & Primer \\
\hline \multirow[t]{2}{*}{$\beta$-actin } & Sense primer: 5'-CGTGAAAAGATGACCCAGAT-3' \\
\hline & Antisense primer: $5^{\prime}$-ACCCTCATAGATGGGCACA-3' \\
\hline \multirow[t]{2}{*}{$N F-\kappa B-p 65$} & Sense primer: 5'-TTCCTGGCGAGAGAAGCAC-3' \\
\hline & Antisense primer: 5'-AAGCTATGGATACTGCGGTCT-3' \\
\hline \multirow[t]{2}{*}{ Caspase 3} & Sense primer: 5'-TACAGCACCTGGTTACTATTC-3' \\
\hline & Antisense primer: 5'-TACAGTTCTTTCGTGAGCAT-3' \\
\hline \multirow[t]{2}{*}{ Caspase 8} & Sense primer: 5'-GACATAACCCAACTCCGAAA-3' \\
\hline & Antisense primer: 5'-TGCAGTGCAGTCGTCGTAA-3' \\
\hline \multirow[t]{2}{*}{ Caspase 9} & Sense primer: 5'-CCGTGGACATTGGTTCTG-3' \\
\hline & Antisense primer: 5'-TCTTGGCAGTCAGGTCGTT-3' \\
\hline \multirow[t]{2}{*}{ Bax } & Sense primer: 5'-TTGCTGATGGCAACTTCAACTG-3' \\
\hline & Antisense primer: 5'-CTTTAGTGCACAGGGCCTTGAG-3' \\
\hline \multirow[t]{2}{*}{$B C l-2$} & Sense primer: 5'-AGGATTGTGGCCTTCTTTGAGTT-3' \\
\hline & Antisense primer: 5'-GCCGGTTCAGGTACTAGTCAT-3' \\
\hline \multirow[t]{2}{*}{ TRIF } & Sense primer: 5'-GGCTAAGGCGTCTCAATCA-3' \\
\hline & Antisense primer: $5^{\prime}-$ TCCCAACACCCATGACAGA-3' \\
\hline \multirow[t]{2}{*}{ IRF-3 } & Sense primer: $5^{\prime}$-CACGCTACACTCTGTGGTTCT-3' \\
\hline & 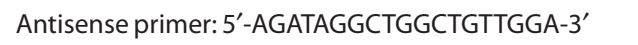 \\
\hline
\end{tabular}

IRF, interferon regulatory factor ; TRIF, Toll/IL-1R domain-containing adaptor-inducing IFN- $\beta$.

performed to achieve maximal downregulation of the $M y D 88$ gene by exposing the cells to dilutions of the viral supernatant in the presence of polybrene ( $9 \mu \mathrm{g} / \mathrm{ml}$; Sigma). Spun the cell culture plates for $30 \mathrm{~min}$ $\left(22^{\circ} \mathrm{C}, 1,200 \mathrm{~g}\right)$ and incubated overnight. Then, the green fluorescent protein positive cells were selected by fluorescence activated cell sorter.

\section{qRT-PCR}

Total RNA was isolated from the intestinal tissue of mice and cultured intestinal epithelium cells, using the RNAiso Plus (TAKARA, Tokyo, Japan) and reverse transcribed ( $1 \mu \mathrm{l}$ of RNA) using the QuantiTect Reverse Transcription Kit (TAKARA). Gene-specific cDNA was amplified and quantified in a real-time thermal cycler system (BioRad, Hercules, CA). PCR amplification was then performed in triplicate. The reaction protocol included preincubation at $95^{\circ} \mathrm{C}$ for $15 \mathrm{~min}$ to activate AmpliTaq Gold DNA Polymerase (Applied Bio-systems, Foster City, CA) and amplification for 40 cycles $\left(15 \mathrm{~s}\right.$ at $95^{\circ} \mathrm{C}, 30 \mathrm{~s}$ at $56^{\circ} \mathrm{C}$, and $60 \mathrm{~s}$ at $\left.72{ }^{\circ} \mathrm{C}\right)$. $\beta$-actin served as internal control. Copy number of target genes (relative to $\beta$-actin) was defined by $2^{-\Delta \Delta \mathrm{Ct}}$.

Specific primers and Taqman probes for MyD88 were designed and synthesized (Invitrogen, Carlsbad, CA) and the sequences are listed in Table 1. The specific primers sequences encoding target genes of NF- $k b$-p65, TRIF, IRF-3, caspase 3, caspase 8, caspase 9, Bcl-2, and Bax are listed in Table 2.

\section{Western Blot}

Total proteins were prepared from the cultured IEC-6-wt and IEC-6MyD88-knockdown cells using Total Protein Extraction Kit (Keygen, Shanghai, China). Protein concentrations were determined using Pierce BCA assay kit (Pierce, Rockford, IL). Twenty micrograms of protein of each sample was loaded on a 10\% SDS-PAGE gel, and proteins that are separated by gel electrophoresis were transferred to polyvinylidene fluoride membranes. The membranes were then incubated at $4{ }^{\circ} \mathrm{C}$ overnight with appropriate primary antibodies diluted in their corresponding blocking buffer. (MyD88: rabbit polyclonal antibody in 1:500 dilution, Abcam, Cambridge, MA; $\beta$-actin: rabbit anti-rat antibody in 1:2,000 dilution, Cell Signaling Technology, Danvers, MA.) After Tris-buffered saline with Tween washing, the membranes were incubated with an HRP-conjugated goat polyclonal antirabbit IgG secondary antibody (Sigma) at a dilution of 1:5,000 After washing, the membranes were analyzed by the enhanced chemiluminescence system (Pierce).

\section{Annexin V for Flow Cytometric Detection of Apoptosis}

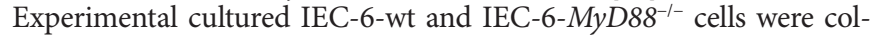
lected to detect apoptosis using the APC Annexin V kit (BD, San Diego, CA) conjunction with a vital dye 7-Amino-Actinomycin (7-AAD; BD). Briefly, $1 \times 10^{5}$ cells were trypsinized (without EDTA), washed twice with cold PBS, and then resuspended in $500 \mu \mathrm{l} 1 \mathrm{X}$ binding buffer. Five microliters of APC Annexin V and $5 \mu$ of 7-AAD were added, gently vortex, and incubated for $15 \mathrm{~min}$ at $25^{\circ} \mathrm{C}$ in the dark. A total of at least 10,000 events were collected and analyzed by flow cytometry (BD) and the apoptotic ratios generated automatically in the Q2 quadrant.

\section{Liquid Chip Multiple Cytokine Assay}

Inflammatory cytokines in intestinal tissue and cell supernatant were detected by Liquid chip, $0.5-1 \mathrm{~g}$ animal tissues from intestinal was treated into $20 \%$ tissue homogenate in $0.86 \%$ cold saline with Ultrasonic Cell Disruption System (OMNI Sonic Ruptor 400W; Omni International, Kennesaw, GA). The tissues homogenate and IEC-6 cell cultural supernatants were centrifuged for $10 \mathrm{~min}$, and the supernatants were collected, stored at $-80^{\circ} \mathrm{C}$ for cytokine analysis on Liquid chip by using Liquid Chip cytokine Kits (R\&D, Minneapolis, $\mathrm{MN}$ ) according to the manufacturer's instructions.

\section{Statistical Analysis}

Data were reported as mean \pm SD and comparisons were made twotailed Student's $t$-test or ANOVA, with statistical significance accepted for $P<0.05$. Additional statistical information regarding specific comparisons was provided in the figure legends.

\section{SUPPLEMENTARY MATERIAL}

Supplementary material is linked to the online version of the paper at http:// www.nature.com/pr

\section{ACKNOWLEDGMENTS}

We thank Laboratory of Stem Cell Biology, West China Hospital, Sichuan University, and Laboratory of Pathology, West China Hospital, Sichuan University, for helpful discussion and expert technical assistance.

\section{STATEMENT OF FINANCIAL SUPPORT}

This work was supported by grants from the National Natural Science Fund of China (NSFC key project no. 30830100; project no. 30972924; project no. 81170439). The project was sponsored by Scientific Research Foundation for the Returned Overseas Chinese Scholars, State Education Ministry (no. 20101174-4-2, Beijing, China) and the Research Fund for the Doctoral Program of Higher Education, State Education Ministry (no. 200806100058 , Beijing, China).

Disclosure: The authors state that they have no conflicts of interest.

\section{REFERENCES}

1. Ryder RW, Shelton JD, Guinan ME. Necrotizing enterocolitis: a prospective multicenter investigation. Am J Epidemiol 1980;112:113-23.

2. Uauy RD, Fanaroff AA, Korones SB, Phillips EA, Phillips JB, Wright LL. Necrotizing enterocolitis in very low birth weight infants: biodemographic and clinical correlates. National Institute of Child Health and Human Development Neonatal Research Network. J Pediatr 1991;119:630-8.

3. Blakely ML, Lally KP, McDonald S, et al.; NEC Subcommittee of the NICHD Neonatal Research Network. Postoperative outcomes of extremely low birth-weight infants with necrotizing enterocolitis or isolated intestinal perforation: a prospective cohort study by the NICHD Neonatal Research Network. Ann Surg 2005;241:984-9; discussion 989-94.

4. Gagliardi L, Bellù R, Cardilli V, De Curtis M; Network Neonatale Lombardo. Necrotising enterocolitis in very low birth weight infants in Italy: incidence and non-nutritional risk factors. J Pediatr Gastroenterol Nutr 2008;47:206-10.

5. Leaphart CL, Cavallo J, Gribar SC, et al. A critical role for TLR4 in the pathogenesis of necrotizing enterocolitis by modulating intestinal injury and repair. J Immunol 2007;179:4808-20. 
6. Gribar SC, Sodhi CP, Richardson WM, et al. Reciprocal expression and signaling of TLR4 and TLR9 in the pathogenesis and treatment of necrotizing enterocolitis. J Immunol 2009;182:636-46.

7. Richardson WM, Sodhi CP, Russo A, et al. Nucleotide-binding oligomerization domain-2 inhibits toll-like receptor-4 signaling in the intestinal epithelium. Gastroenterology 2010, 139: 904-917e6.

8. Sodhi CP, Shi XH, Richardson WM, et al. Toll-like receptor-4 inhibits enterocyte proliferation via impaired beta-catenin signaling in necrotizing enterocolitis. Gastroenterology 2010;138:185-96.

9. Sodhi CP, Neal MD, Siggers R, et al. Intestinal epithelial Toll-like receptor 4 regulates goblet cell development and is required for necrotizing enterocolitis in mice. Gastroenterology 2012;143:708-18.e1-5.

10. Afrazi A, Sodhi CP, Richardson W, et al. New insights into the pathogenesis and treatment of necrotizing enterocolitis: Toll-like receptors and beyond. Pediatr Res 2011;69:183-8.

11. Yamamoto M, Sato S, Mori K, et al. Cutting edge: a novel Toll/IL-1 receptor domain-containing adapter that preferentially activates the IFN-beta promoter in the Toll-like receptor signaling. J Immunol 2002;169:6668-72.

12. Larsson E, Tremaroli V, Lee YS, et al. Analysis of gut microbial regulation of host gene expression along the length of the gut and regulation of gut microbial ecology through MyD88. Gut 2012;61:1124-31.

13. Castoldi A, Braga TT, Correa-Costa M, et al. TLR2, TLR4 and the MYD88 signaling pathway are crucial for neutrophil migration in acute kidney injury induced by sepsis. PLoS ONE 2012;7:e37584.

14. Tchaptchet S, Gumenscheimer M, Kalis C, et al. TLR9-dependent and independent pathways drive activation of the immune system by Propionibacterium acnes. PLoS ONE 2012;7:e39155.

15. Ciorba MA, Riehl TE, Rao MS, et al. Lactobacillus probiotic protects intestinal epithelium from radiation injury in a TLR-2/cyclo-oxygenase-2-dependent manner. Gut 2012;61:829-38.

16. Ohnishi $\mathrm{H}$, Tochio $\mathrm{H}$, Kato $\mathrm{Z}$, et al. TRAM is involved in IL-18 signaling and functions as a sorting adaptor for MyD88. PLoS ONE 2012;7:e38423.

17. Reis J, Hassan F, Guan XQ, et al. The immunoproteasomes regulate LPS induced TRIF/TRAM signaling pathway in murine macrophages. Cell Biochem Biophys 2011;60:119-26.

18. Oshiumi H, Sasai M, Shida K, Fujita T, Matsumoto M, Seya T. TIR-containing adapter molecule (TICAM)-2, a bridging adapter recruiting to toll-like receptor 4 TICAM-1 that induces interferon-beta. J Biol Chem 2003;278:49751-62.

19. Fitzgerald KA, Rowe DC, Barnes BJ, et al. LPS-TLR4 signaling to IRF-3/7 and NF-kappaB involves the toll adapters TRAM and TRIF. J Exp Med 2003;198:1043-55.

20. Clark K, Takeuchi O, Akira S, Cohen P. The TRAF-associated protein TANK facilitates cross-talk within the IkappaB kinase family during Tolllike receptor signaling. Proc Natl Acad Sci USA 2011;108:17093-8.

21. Ogasawara N, Sasaki M, Itoh Y, et al. Rebamipide suppresses TLR-TBK1 signaling pathway resulting in regulating IRF3/7 and IFN-a/ß reduction. J Clin Biochem Nutr 2011;48:154-60.

22. Fornarino S, Laval G, Barreiro LB, Manry J, Vasseur E, QuintanaMurci L. Evolution of the TIR domain-containing adaptors in humans: swinging between constraint and adaptation. Mol Biol Evol 2011;28:3087-97.

23. Barnes PJ, Karin M. Nuclear factor-kappaB: a pivotal transcription factor in chronic inflammatory diseases. N Engl J Med 1997;336: 1066-71.

24. Liu Y, Fatheree NY, Mangalat N, Rhoads JM. Lactobacillus reuteri strains reduce incidence and severity of experimental necrotizing enterocolitis via modulation of TLR4 and NF-?B signaling in the intestine. Am J Physiol Gastrointest Liver Physiol 2012;302:G608-17.

25. Deghmane AE, El Kafsi H, Giorgini D, Abaza A, Taha MK. Late repression of NF-?B activity by invasive but not non-invasive meningococcal isolates is required to display apoptosis of epithelial cells. PLoS Pathog 2011;7:e1002403.

26. Hackam DJ, Upperman JS, Grishin A, Ford HR. Disordered enterocyte signaling and intestinal barrier dysfunction in the pathogenesis of necrotizing enterocolitis. Semin Pediatr Surg 2005;14:49-57.

27. Li JH, D’Alessio A, Pober JS. Lipopolysaccharide can trigger a cathepsin B-dependent programmed death response in human endothelial cells. Am J Pathol 2009;175:1124-35.

28. Tamura R, Kanda T, Imazeki F, et al. Hepatitis C Virus nonstructural 5A protein inhibits lipopolysaccharide-mediated apoptosis of hepatocytes by decreasing expression of Toll-like receptor 4. J Infect Dis 2011;204:793801.

29. Li Y, Si R, Feng Y, et al. Myocardial ischemia activates an injurious innate immune signaling via cardiac heat shock protein 60 and Toll-like receptor 4. J Biol Chem 2011;286:31308-19.

30. Ko MK, Saraswathy S, Parikh JG, Rao NA. The role of TLR4 activation in photoreceptor mitochondrial oxidative stress. Invest Ophthalmol Vis Sci 2011;52:5824-35.

31. Fukata M, Chen A, Klepper A, et al. Cox-2 is regulated by Toll-like receptor-4 (TLR4) signaling: Role in proliferation and apoptosis in the intestine. Gastroenterology 2006;131:862-77.

32. He S, Liang Y, Shao F, Wang X. Toll-like receptors activate programmed necrosis in macrophages through a receptor-interacting kinase-3-mediated pathway. Proc Natl Acad Sci USA 2011;108:20054-9.

33. Khailova L, Mount Patrick SK, Arganbright KM, Halpern MD, Kinouchi T, Dvorak B. Bifidobacterium bifidum reduces apoptosis in the intestinal epithelium in necrotizing enterocolitis. Am J Physiol Gastrointest Liver Physiol 2010;299:G1118-27.

34. Clark JA, Lane RH, Maclennan NK, et al. Epidermal growth factor reduces intestinal apoptosis in an experimental model of necrotizing enterocolitis. Am J Physiol Gastrointest Liver Physiol 2005;288:G755-62.

35. Kawai T, Adachi O, Ogawa T, Takeda K, Akira S. Unresponsiveness of MyD88-deficient mice to endotoxin. Immunity 1999;11:115-22.

36. Johnson AC, Li X, Pearlman E. MyD88 functions as a negative regulator of TLR3/TRIF-induced corneal inflammation by inhibiting activation of c-Jun N-terminal kinase. J Biol Chem 2008;283:3988-96.

37. Nadler EP, Dickinson E, Knisely A, et al. Expression of inducible nitric oxide synthase and interleukin-12 in experimental necrotizing enterocolitis. J Surg Res 2000;92:71-7. 